\title{
Framework to predict optimal buffer layer pairing for thin film solar cell absorbers: A case study for tin sulfide/zinc oxysulfide
}

\section{Citation}

Mangan, Niall M., Riley E. Brandt, Vera Steinmann, R. Jaramillo, Chuanxi Yang, Jeremy R. Poindexter, Rupak Chakraborty, et al. 2015. "Framework to Predict Optimal Buffer Layer Pairing for Thin Film Solar Cell Absorbers: A Case Study for Tin Sulfide/zinc Oxysulfide." Journal of Applied Physics 118 (11) (September 21): 115102. Portico. doi:10.1063/1.4930581.

\section{Published Version}

doi:10.1063/1.4930581

\section{Permanent link}

http://nrs.harvard.edu/urn-3:HUL.InstRepos:25659035

\section{Terms of Use}

This article was downloaded from Harvard University's DASH repository, and is made available under the terms and conditions applicable to Open Access Policy Articles, as set forth at http:// nrs.harvard.edu/urn-3:HUL.InstRepos:dash.current.terms-of-use\#OAP

\section{Share Your Story}

The Harvard community has made this article openly available.

Please share how this access benefits you. Submit a story.

Accessibility 


\title{
Framework to predict optimal buffer layer pairing for thin film solar cell absorbers: A case study for tin sulfide/zinc oxysulfide
}

\author{
Niall M. Mangan, ${ }^{1}$ Riley E. Brandt,${ }^{1}$ Vera Steinmann, ${ }^{1}$ R. Jaramillo, ${ }^{1}$ Chuanxi Yang, ${ }^{2}$ Jeremy R. \\ Poindexter, ${ }^{1}$ Rupak Chakraborty, ${ }^{1}$ Helen Hejin Park, ${ }^{2}$ Xizhu Zhao, ${ }^{2}$ Roy G. Gordon, ${ }^{2}$ and Tonio Buonassisi ${ }^{1}$ \\ ${ }^{1}$ Massachusetts Institute of Technology, Cambridge, Massachusetts 02139, USA \\ ${ }^{2}$ Harvard University, Cambridge, Massachusetts 02139, USA
}

\begin{abstract}
An outstanding challenge in the development of novel functional materials for optoelectronic devices is identifying suitable charge-carrier contact layers. Herein, we simulate the photovoltaic device performance of various $n$-type contact material pairings with tin(II) sulfide (SnS), a $p$-type absorber. The performance of the contacting material, and resulting device efficiency, depend most strongly on two variables: conduction band offset between absorber and contact layer, and doping concentration within the contact layer. By generating a 2D contour plot of device efficiency as a function of these two variables, we create a performance-space plot for contacting layers on a given absorber material. For a simulated high-lifetime SnS absorber, this 2D performance-space illustrates two maxima, one local and one global. The local maximum occurs over a wide range of contact-layer doping concentrations (below $10^{16} \mathrm{~cm}^{-3}$ ), but only a narrow range of conduction band offsets $(0$ to $-0.1 \mathrm{eV})$, and is highly sensitive to interface recombination. This first maximum is ideal for early-stage absorber research because it is more robust to low bulk-minority-carrier lifetime and pinholes (shunts), enabling device efficiencies approaching half the Shockley-Queisser limit, greater than $16 \%$. The global maximum is achieved with contact-layer doping concentrations greater than $10^{18} \mathrm{~cm}^{-3}$, but for a wider range of band offsets $(-0.1$ to $0.2 \mathrm{eV})$, and is insensitive to interface recombination. This second maximum is ideal for high-quality films because it is more robust to interface recombination, enabling device efficiencies approaching the Shockley-Queisser limit, greater than $20 \%$. Band offset measurements using X-ray photoelectron spectroscopy and carrier concentration approximated from resistivity measurements are used to characterize the zinc oxysulfide contacting layers in recent record-efficiency SnS devices. Simulations representative of these present-day devices suggest that record efficiency $\mathrm{SnS}$ devices are optimized for the second local maximum, due to low absorber lifetime and relatively well passivated interfaces. By employing contact layers with higher carrier concentrations and lower electron affinities, a higher efficiency ceiling can be enabled.
\end{abstract}




\section{INTRODUCTION:}

For many thin film photovoltaic materials, a major hurdle to achieving high efficiency is finding the appropriate $n$-type material pairings for making optimized heterojunctions. Sub-optimal material pairings can cause either current blocking, limiting short-circuit current $\left(J_{\mathrm{SC}}\right)$, or large interface recombination currents, limiting open-circuit voltage $\left(V_{\mathrm{OC}}\right)$. Studies in commercially successful $p$-type thin film absorber materials, such as CIGS and CdTe, have shown that the optimal $n$-type material pairing for a thin-filmheterojunction has the following characteristics [1, 2]: wide band gap, large valence band offset, high carrier concentration, and spike-type(positive) conduction band offset. While these studies provide design guidelines optimizing the electronic properties of a material pairing, the exact values for co-optimization of carrier concentration and conduction band offset are highly dependent on the absorber material electronic properties.

An outstanding problem in this field is to provide a standard framework to visualize the performance across these parameters. To address this challenge we model the impact of these parameters and direct device development as absorber lifetime improves. As an example, this framework is applied to tin(II) sulfide (SnS) devices. We develop a model for a feasible, but as of yet unrealized, high efficiency SnS device and use the model to map out device performance for varying carrier concentration and band alignment of contact layers. We then perform the same analysis on a $\mathrm{SnS}$ device representative of our present-day device performance.

SnS has promising optical [3] and electronic properties [4, 5] for a photovoltaic absorber material, is Earth-abundant, and easily manufacturable [6]. Much experimental effort has been put into finding an optimal contact layer for $\mathrm{SnS}$, as summarized in Ref. 7]. However, SnS devices have only recently achieved NREL certified efficiencies of $4.36 \%$ for atomic layer deposited SnS [8], and 3.88\% for thermally evaporated SnS [6], falling far short of the Shockley-Queisser efficiency limit of $31 \%$ [9. The devices investigated here use a $\mathrm{Mo} / \mathrm{SnS} / \mathrm{Zn}(\mathrm{O}, \mathrm{S}) / \mathrm{ZnO} / \mathrm{ITO} / \mathrm{Ag}$ heterojunction device stack 10. Molybdenum (Mo) is the back contact, $\mathrm{SnS}$ is a $p$-type absorber material, zinc oxysulfide $\mathrm{Zn}(\mathrm{O}, \mathrm{S})$ is the $n$-type buffer layer or contact layer of interest, zinc-oxide $(\mathrm{ZnO})$ is an $n$-type window layer, indium tin oxide (ITO) is an $n$-type transparent conducting oxide, and $\mathrm{Ag}$ is a metal contact. Here, we focus on performance due to varying the electric properties, specifically the conduction band-offset and carrier concentration, of the buffer layer, $\operatorname{Zn}(\mathrm{O}, \mathrm{S})$.

By varying the sulfur to zinc composition during atomic layer deposition of the $\mathrm{Zn}(\mathrm{O}, \mathrm{S})$ buffer layer, the electron affinity, and therefore conduction band alignment with $\mathrm{SnS}$ can be tuned [11, 12. Additionally, nitrogen doping of the $\mathrm{Zn}(\mathrm{O}, \mathrm{S})$ lowers the electron carrier concentration in the buffer layer. Experimental optimization following [8, 13, found that the highest performing device had a S/Zn ratio of 0.14 and nitrogen doping. Measurements of the carrier concentration under illumination (to account for photoconductivity effects) and band alignment are performed for $\mathrm{Zn}(\mathrm{O}, \mathrm{S})$ with varying $\mathrm{S} / \mathrm{Zn}$ composition, including the composition resulting in record device efficiencies $[6$, 8 . These experimentally achievable compositions are contextualized within the performance space defined by simulation. The simulated efficiencies are compared with experimental results for devices using a range of buffer layer compositions. The simulated performance space shows there are still efficiency gains to be had by optimizing the buffer layer with present-day lifetimes, within the range of buffer layer carrier concentrations and conduction band offsets experimentally achievable by the $\mathrm{Zn}(\mathrm{O}, \mathrm{S})$ system. The performance spaces also provide guidance for re-optimizing the buffer layer to one with a higher carrier concentration as the $\mathrm{SnS}$ bulk lifetime improves. Similar performance spaces could be simulated for other absorber systems, demystifying the choice of contact layer pairing and reducing the number of devices that must be fabricated.

We simulate these thin-film photovoltaic devices in SCAPS 1-D, which self consistently solves the drift-diffusion equations for electrons and holes and Poisson's equation for the electric field [14. Definition files for our simulation can be found in the supplementary material. SCAPS is required to reproduce our simulations and can be obtained for free from Ghent University. Batch simulations results from SCAPS are further processed an analyzed in MATLAB. Here, we detail our assumptions for the parameters in our device simulations, except for the buffer layer carrier concentration and conduction band offset which are varied. There are two scenarios defined by a set of parameters: the first scenario represents potential high-efficiency SnS devices, and the second scenario represents present-day SnS devices. The parameters for the device stack presented here vary from those assumed in previous simulation of SnS devices [15. A schematic of the device stack is shown in Figure 2, and parameters defining this device in SCAPS are summarized in Table 1 . Justification for the choice of some parameters are detailed here.

Although SnS has a direct and indirect transitions that influence optoelectronic properties, the indirect transition at $1.1 \mathrm{eV}$ is the relevant transition for defect-assisted recombination [3]. For our baseline simulation we use an average 


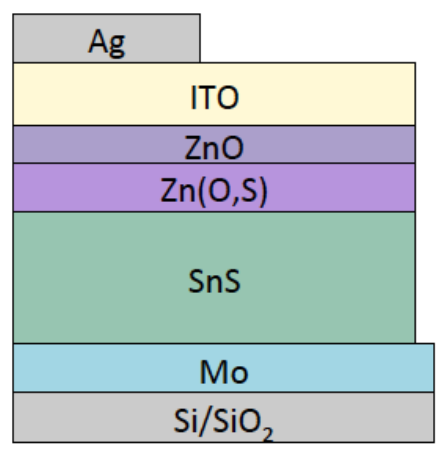

FIG. 1. Experimental device stack for SnS-based devices, including silver contact, indium tin oxide and zinc-oxide transparent contact/window layers, zinc-oxysulfide buffer layer, tin-sulfide absorber layer, and molybdenum back contact. The stack is grown on a silicon substrate with a silicon-oxide layer.

\begin{tabular}{|c|c|c|c|}
\hline \multirow[t]{2}{*}{ Material Parameter } & \multicolumn{2}{|c|}{ Value for } & \multirow[t]{2}{*}{ Source } \\
\hline & present-day scenario & high-efficiency scenario & \\
\hline SnS thickness & \multicolumn{2}{|c|}{$1 \mu \mathrm{m}$} & SEM cross-section \\
\hline SnS band gap & \multicolumn{2}{|c|}{$1.1 \mathrm{eV}$} & 3 \\
\hline SnS minority carrier lifetime & $100 \mathrm{ps}$ & $500 \mathrm{~ns}$ & see Methods, [16 18 \\
\hline SnS static dielectric constant & \multicolumn{2}{|c|}{40} & 19 \\
\hline SnS $h^{+}\left(e^{-}\right)$mobility & \multicolumn{2}{|c|}{$30(74) \mathrm{cm}^{2} /(\mathrm{Vs})$} & $\begin{array}{l}\text { Hall effect measurement } \\
\left(e^{-} \text {calculated using } m_{\mathrm{h}} / m_{\mathrm{e}} \text { from }\right. \\
{[3]}\end{array}$ \\
\hline $\mathrm{SnS} h^{+}$carrier density & \multicolumn{2}{|c|}{$10^{16} \mathrm{~cm}^{-3}$} & Hall effect measurement \\
\hline $\operatorname{SnS} N_{\mathrm{C}}\left(N_{\mathrm{V}}\right)$ density of states & \multicolumn{2}{|c|}{$3.6 \times 10^{18}\left(1.4 \times 10^{19}\right) \mathrm{cm}^{-3}$} & $\begin{array}{l}\text { calculated from effective masses } \\
{[3}\end{array}$ \\
\hline ITO, $\mathrm{ZnO}, \mathrm{Zn}(\mathrm{O}, \mathrm{S})$ band gaps & \multicolumn{2}{|c|}{$>2.5 \mathrm{eV}$} & see Methods \\
\hline $\begin{array}{l}\mathrm{SnS} / \mathrm{Zn}(\mathrm{O}, \mathrm{S}) \text { interface recombi- } \\
\text { nation velocity }\end{array}$ & \multicolumn{2}{|c|}{$2 \times 10^{3} \mathrm{~cm} / \mathrm{s}$} & see Fig. 2 \\
\hline $\mathrm{Zn}(\mathrm{O}, \mathrm{S})$ thickness & \multicolumn{2}{|c|}{$36 \mathrm{~nm}$} & 20 \\
\hline $\mathrm{Zn}(\mathrm{O}, \mathrm{S}), \mathrm{ZnO}$ dielectric constant & \multicolumn{2}{|c|}{9} & 21 \\
\hline $\begin{array}{l}\mathrm{Zn}(\mathrm{O}, \mathrm{S}), \mathrm{ZnO} N_{\mathrm{C}}\left(N_{\mathrm{V}}\right) \text { density of } \\
\text { states }\end{array}$ & \multicolumn{2}{|c|}{$2 \times 10^{18}\left(7 \times 10^{19}\right) \mathrm{cm}^{-3}$} & $\begin{array}{l}\text { calculated from effective masses } \\
22\end{array}$ \\
\hline $\mathrm{Zn}(\mathrm{O}, \mathrm{S}) / \mathrm{ZnO}$ band offset & \multicolumn{2}{|c|}{$0 \mathrm{eV}$} & 11 for $\mathrm{S} / \mathrm{Zn}=0.14 \mathrm{Zn}(\mathrm{O}, \mathrm{S})$ \\
\hline ZnO thickness & \multicolumn{2}{|c|}{$18 \mathrm{~nm}$} & 20 \\
\hline $\mathrm{ZnO} e-$ carrier density & \multicolumn{2}{|c|}{$10^{19} \mathrm{~cm}^{-3}$} & 13,23 \\
\hline $\mathrm{ZnO}, \mathrm{Zn}(\mathrm{O}, \mathrm{S})$, ITO lifetime & $<10^{-2} \mathrm{~ns}$ & no loss & $\begin{array}{l}\text { assuming no collection in these } \\
\text { layers [ 6] }\end{array}$ \\
\hline Optical absorption for all layers & wavelength dependent & $\begin{array}{l}\text { no absorption in buffer } \\
\text { or window layers }\end{array}$ & 6 \\
\hline ITO and Mo contacts & \multicolumn{2}{|c|}{ flat-band alignment (ohmic) } & 24 \\
\hline $\begin{array}{l}\text { Recombination velocity at con- } \\
\text { tacts }\end{array}$ & $10^{7} \mathrm{~cm} / \mathrm{s}$ & $10^{2} \mathrm{~cm} / \mathrm{s}$ & high limit/ no loss \\
\hline
\end{tabular}

TABLE I. Parameters held fixed in all simulations are summarized here. Carrier concentration in $\mathrm{Zn}(\mathrm{O}, \mathrm{S})$ and band offset between $\mathrm{SnS}$ and $\mathrm{Zn}(\mathrm{O}, \mathrm{S})$, are varied as indicated in the text to produce the buffer layer performance space. Split column indicates when values are different for the current and high performance SnS device simulations. Other parameters defining the simulations in SCAPS can be found in the device definition files included in the supplementary materials.

of the static dielectric constant across different orientations [19, 25]. SnS hole mobility is obtained from Hall effect measurements. The SnS electron mobility is calculated as $\mu_{e}=\mu_{h} \frac{m_{h}^{*}}{m_{e}^{*}}$, where the effective mass ratio between electrons 
and holes is averaged across orientations as $\frac{m_{h}}{m_{e}}=\frac{0.68}{0.28}=2.46[3]$. Previous QE analysis suggests that the minority carrier lifetime for the present-day scenario is approximately $100 \mathrm{ps}$ [16. Recently this order of magnitude was verified by terahertz pump-probe free carrier absorption spectroscopy [17, 18. The high-efficiency scenario assumes a SnS lifetime of $500 \mathrm{~ns}$.

We use measured values for absorption in $\mathrm{SnS}$ as are reported in Ref. 6. The same study shows that all carriers generated in the window and buffer layers are lost and not collected in present-day devices. To enforce this we assume lifetimes of $<10^{-2} \mathrm{~ns}$ for the present-day scenario. For the high-efficiency scenario we assume no generation (the absorption coefficient is set to zero) or recombination in the buffer $(\mathrm{Zn}(\mathrm{O}, \mathrm{S}))$ and window $(\mathrm{ZnO} / \mathrm{ITO})$ layers. In the present-day scenario the absorption data for the $\mathrm{ZnO}$ and ITO from Ref. 6] are used, accurately modeling the optical band-gap of the material.

In both the present-day and high-efficiency scenarios, the electronic band gaps of the ZnO and ITO front layers do not affect generation or recombination. We therefore define the ITO, $\mathrm{ZnO}$ and $\mathrm{Zn}(\mathrm{O}, \mathrm{S})$ electronic band gaps to be 2.5 $\mathrm{eV}$ to enable numerical convergence. These gaps are sufficiently large enough to block hole transport from the SnS to the buffer layer. Reducing the band gap from the physical values (up to $3.3 \mathrm{eV}$ ) down to $2.5 \mathrm{eV}$ reduces dramatic variations in minority-carrier concentration, enabling numerical convergence with no impact on device performance.

The electrical parameters of the $\mathrm{ZnO}$ window layer are held constant as shown in Table I. For $\mathrm{ZnO}$, the values for carrier concentration and permittivity come from [23] and the carrier concentration values are consistent with measurements on $100 \mathrm{~nm}$ thick films [13. We also use an average of the $\mathrm{ZnO}$ effective masses in Ref. 22 to calculate the effective density of states in $\mathrm{ZnO}$. Rather than assuming absolute electron affinities from the literature for each layer, all band offsets are treated as relative. There is zero conduction band offset between $\mathrm{ZnO}$ and $\mathrm{Zn}(\mathrm{O}, \mathrm{S})$ for $\mathrm{S} / \mathrm{Zn}$ $=0.14$ 11, and we set the conduction band offset between $\mathrm{ZnO}$ and $\mathrm{Zn}(\mathrm{O}, \mathrm{S})$ for all other $\mathrm{S} / \mathrm{Zn}$ compositions relative to this case. The $\mathrm{Zn}(\mathrm{O}, \mathrm{S})$ conduction band-offset from $\mathrm{SnS}$ for $\mathrm{S} / \mathrm{Zn}=0.14$ is measured using X-ray photoelectron spectroscopy.

The parameters for ITO do not affect the simulation, and ITO acts as an ohmic contact on ZnO. We include it in the device stack to correctly account for optical and electronic losses in the ITO for the present-day scenario. Similarly, the silver contact does not impact simulations The built-in voltage is set by the $\mathrm{SnS}, \mathrm{Zn}(\mathrm{O}, \mathrm{S})$, and $\mathrm{ZnO}$ layers, as long as $\mathrm{ZnO}$ is not fully depleted. Given carrier concentrations of $10^{19} \mathrm{~cm}^{-3}$ and $18 \mathrm{~nm}$ thickness, $\mathrm{ZnO}$ will not be depleted.

The molybdenum (Mo) back contact is ohmic with $\mathrm{SnS}$, as demonstrated by transmission line contact resistance measurements 24. The recombination velocity at the Mo back contact is assumed to be high, $10^{7} \mathrm{~cm} / \mathrm{s}$, in the present-day scenario and low $10^{2} \mathrm{~cm} / \mathrm{s}$ for the high-efficiency scenario. Such low surface recombination velocities have been achieved using metal-oxide passivated contacts in other systems [26, 27.

\section{B. Fitting to obtain interface recombination velocity}

The interface recombination velocity for the $\operatorname{SnS} / \mathrm{Zn}(\mathrm{O}, \mathrm{S})$ interface is unknown. To determine a reasonable value for interface recombination, we simulate the current-voltage and quantum efficiency over the experimentally determined range of buffer layer carrier concentration and band offset. We vary the interface recombination velocity between $10^{2}$ $\mathrm{cm} / \mathrm{s}$ and $10^{7} \mathrm{~cm} / \mathrm{s}$. The results of this full parameter sweep on current-voltage and quantum efficiency are shown in light grey in Figure 2 and compared with experimental results for a device with $\mathrm{S} / \mathrm{Zn}=0.14$ and nitrogen doping (dashed line). We then filter these simulated curves for those that have $V_{\mathrm{OC}}=0.32 \pm 0.02 \mathrm{~V}$. This reduced range of simulations is plotted in darker grey on Figure $2 \mathrm{a}$. As is shown by the limited variation of $J_{\mathrm{SC}}$ (Figure $2 \mathrm{a}$ ) and quantum efficiency (Figure 2 $\mathrm{p}$ ) over the full range of interface velocities (light grey curves), $J_{\mathrm{SC}}$ and quantum efficiency are set primarily by bulk lifetime and do not effectively constrain the buffer-layer parameters. The differences in fit between simulation and experiment are small given that most parameters are order-of-magnitude estimates.

While the interface recombination velocity fit is not unique, it only varies by an order of magnitude within the error bars of measured conduction band offset. For larger interface recombination velocities the best-fit conduction band offset must be closer to zero as shown in the inset of Figure 2 a. Both increasing interface recombination velocity and increasingly negative conduction band offsets will increase interface recombination. The buffer layer carrier concentration does not affect the performance or fit in this range. An interface velocity of $2 \times 10^{3} \mathrm{~cm} / \mathrm{s}$ fits well for the measured conduction band offset of $-0.38 \mathrm{eV}$, so we use this value in all simulations.

\section{Experimental Measurements}

Carrier concentrations of thin $\mathrm{Zn}(\mathrm{O}, \mathrm{S})$ buffer layers were determined through a combination of Hall effect and sheet resistance measurements. Hall effect measurements were performed on thin film samples ranging from 30-100 
a

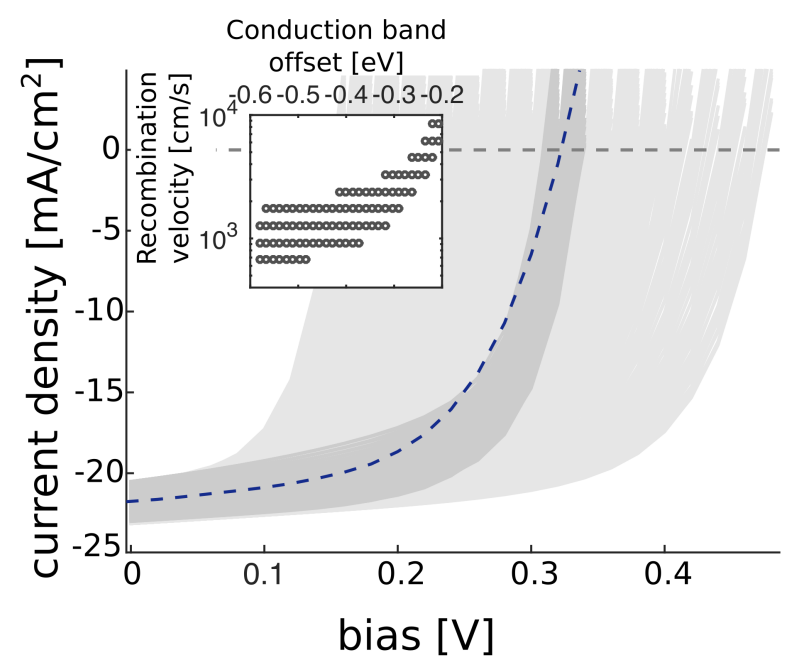

b

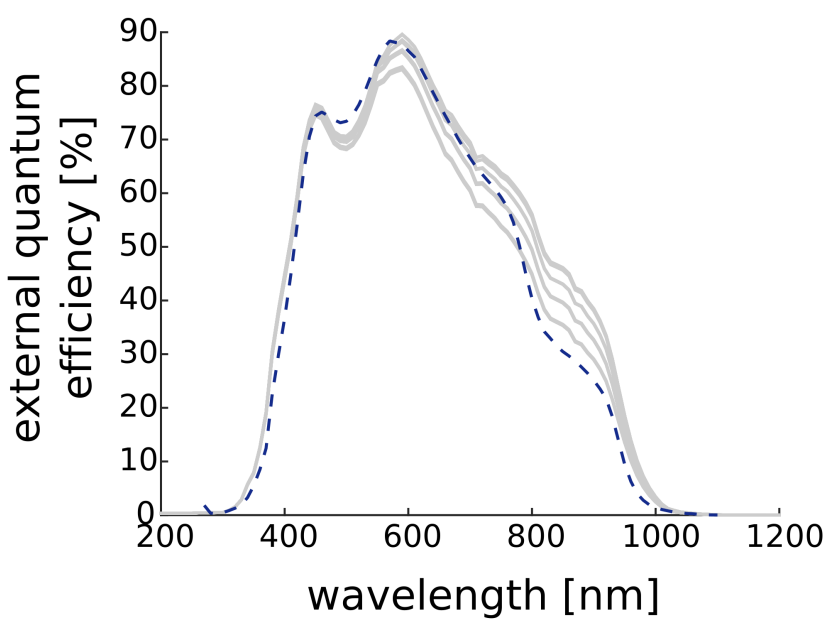

$\mathrm{nm}$, under dark conditions, with magnetic fields ranging from -1.5 to $+1.5 \mathrm{~T}$. In addition, thin samples of 30-40 $\mathrm{nm}$ thickness were measured using an AC Hall effect measurement with a rotating magnet system 28. The sheet resistance was measured on device-thickness films $(38 \mathrm{~nm})$ using a two-point contact and an electrometer with a 200 G $\Omega$ input impedance. We illuminate the sample with AM1.5 light for approximately one hour, allowing the resistivity to stabilized. We then shut off the light and measure the resistivity for 14 hours (See SI). We report illuminated values, as these values are representative of solar cell operation. Using the electron mobility determined by Hall effect, thickness, and the resistivity data we estimate the carrier concentration.

Band offset measurement methods are presented in the supplemental material.

FIG. 2. Comparison of current-voltage (A) and quantum efficiency (B) measurements (dashed line) with simulation (grey-solid) for devices with $\mathrm{Zn}(\mathrm{O}, \mathrm{S}), \mathrm{S} / \mathrm{Zn}=0.14$, and nitrogen doping. Light grey simulated curves in both $\mathrm{A}$ and $\mathrm{B}$ show the range of behaviors over experimentally measured error in buffer carrier concentration and band offset shown in Figure $4 \mathrm{~b}$ and c. This grey area also includes the performance for interface recombination velocities varying from $10^{2}$ to $10^{7} \mathrm{~cm} / \mathrm{s}$. Curves with $V_{\mathrm{OC}}=0.32 \pm 0.02 \mathrm{~V}$ are shown in dark grey in (A). The recombination velocity and conduction band offset for this subset of curves are correlated, as shown in the inset. Circles represent the parameters for an individual simulation.

\section{RESULTS:}

\section{A. Buffer layer performance space for high-efficiency scenario}

To fully visualize the effect of buffer layer parameters for the high-efficiency scenario, we simulate current-voltage curves for SnS devices with varying buffer layer compositions. Figure 3 shows a contour plot of the resulting device efficiency as a function of buffer layer carrier concentration and conduction band offset. To separate out the impact of the buffer layer from other loss mechanisms, we assume an SnS minority carrier lifetime of 500 ns and no optical or recombination losses in the buffer, window, or contacting layers. Details of the device parameters can be found in the methods section. The respective current-voltage curves and band diagrams are plotted for representative points (A-E) marked on the contour plot.

The contour plot in Figure 3 demonstrates a range of different device behaviors due to the buffer layer pairing. The highest-efficiency regime in the upper right (A) has high carrier concentration $>10^{18} \mathrm{~cm}^{-3}$ in the buffer layer and a conduction-band offset between -0.2 and $0.2 \mathrm{eV}$. In the high-efficiency region, the high number of electrons in the buffer layer sets up a larger depletion region in the absorber, moving the location of equal hole and electron populations (the $p-n$ junction) into the absorber and away from the physical $\mathrm{SnS} / \mathrm{Zn}(\mathrm{O}, \mathrm{S})$ interface. The depletion of holes at the interface is evident due to the large gap between the hole quasi-Fermi level and the valence band at 

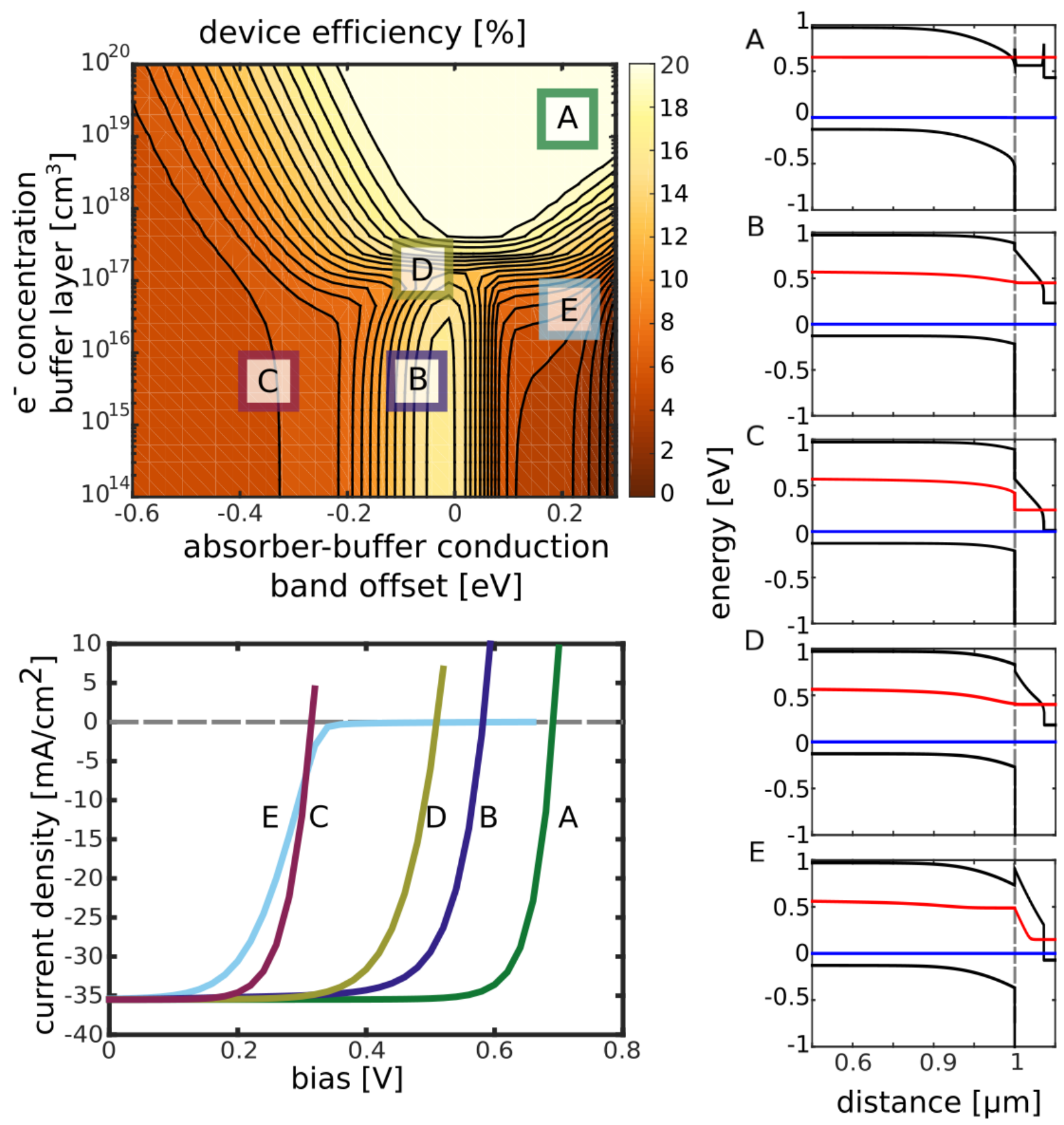

FIG. 3. Contact-layer performance space for $\mathrm{Zn}(O, S)$ buffer layers in SnS devices with high lifetime: Colormap of simulated efficiency (color intensity) of $\mathrm{SnS}$ device with varying conduction band offset between absorber and buffer layer (x-axis) and varying buffer layer carrier concentration (y-axis). This simulation is for a 500 ns lifetime SnS absorber with no optical or recombination losses from the buffer, window or contacting layers. Color indicates high (lighter) and low (darker) efficiency from 0 to 20\%. Parameters for the device stack, other than the band offset and buffer layer carrier concentration, are found in the methods section. Current-voltage behavior and band alignment for points A through E indicated on the counter plot are also shown. The numerically calculated band diagrams show the conduction and valence bands in black, illustrating the respective band offset and band bending between $\mathrm{SnS}$ and the buffer layer at the max power point. The electron (red) and hole (blue) quasi-Fermi levels are also illustrated. These band diagrams are zoomed in to the junction and do not show the full thickness of SnS and ITO. 
the interface in the band diagram for location (A). The reduction of holes at the interface, and movement of the junction into the absorber reduces the interface recombination. When buffer carrier concentrations are $10^{17} \mathrm{~cm}^{-3}$, the electron quasi-Fermi level moves toward mid-gap at the interface, the $p$-n junction is at the physical $\operatorname{SnS} / \operatorname{Zn}(\mathrm{O}, \mathrm{S})$ interface, and interface recombination is maximized 1, 2, 29, Thus, a device at location (D) has much larger interface recombination than devices with higher (A) or lower (B) carrier concentration.

At carrier concentrations less than $10^{-18} \mathrm{~cm}^{-3}$ and conduction-band offsets greater than zero (E), performance drops off due to a current-blocking spike in the conduction band. Comparing the band diagrams for a device with this current-blocking spike (E) and the highest-efficiency regime (A), we can see that both have spike-type offsets. In the high efficiency device (A), the high buffer-layer carrier concentration increases band bending allowing unimpeded thermionic electron transport ,as indicated by the location of the electron quasi-fermi level above the conduction band. In the case of lower carrier concentration (E), the electron quasi-Fermi level is far from the conduction band in the buffer layer and transport will be impeded. These limiting behaviors have been described previously [1], but their onset as a function of carrier concentration and conduction band offset are more readily visualized in a 2-D plot.

Another interesting aspect revealed by this parameter-space plot, is that there is a local maximum for small cliff offset $(-0.1 \mathrm{eV})$ and low carrier concentration $<10^{16} \mathrm{~cm}^{-3}$ (B). A local maximum such as this could be mistaken for a global maximum during experimental optimization. Additionally, to move from this local maximum (B) to the high carrier concentration maximum (A), one must pass though a region of lower device performance. Experimentalists should not be discouraged by lower device performance, as further changes to the carrier concentration and offset will result in higher efficiency. Taken together, this full picture provides a roadmap for developing SnS buffer-layer pairings. Next, we address how the buffer layer performance space changes for present-day scenario, and where the highest performing buffer layer falls in this space.

\section{B. Analysis of buffer layer performance space for present-day scenario}

We revise the numerical SCAPS model to represent present-day performance of SnS devices. After including lower minority-carrier lifetime, optical losses, and contact losses representative of present-day devices (see Methods for details), the buffer-layer performance space changes (Figure $4 \mathrm{a}$ ). The buffer layer with maximum efficiency is now one with a small cliff conduction band offset $(-0.1 \mathrm{eV})$ with low carrier concentration $\left(<10^{16} \mathrm{~cm}^{-2}\right)$. Additionally, the efficiency decreases as the conduction band offset varies from a $-0.4 \mathrm{eV}$ cliff to a $0.3 \mathrm{eV}$ spike conduction band offset for carrier concentrations above $10^{18} \mathrm{~cm}^{-3}$. In this region, the gains seen in Figure 3 for band alignment closer to zero are masked by bulk recombination. Increased interface recombination when the Fermi-level sits mid-gap, and current blocking for low carrier concentration buffer layers with spike-type offsets still define the lower carrier concentration region.

We experimentally characterize the carrier concentration and conduction-band offset for buffer layers of varying compositions, and overlay the results on Figure 4 4 . The carrier concentration with $\mathrm{S} / \mathrm{Zn}=0.14$ (with and without nitrogen doping), 0.26, and 0.37 are inferred from resistivity and Hall effect measurements (See Methods for details). We measure films of active layer thickness $(38 \mathrm{~nm})$ under illumination to account for thickness and photoconductivity effects. Increased carrier concentration in the light is not necessarily a problem for device performance $[30+32]$. Both the $\mathrm{S} / \mathrm{Zn}=0.14$ with nitrogen doping and $\mathrm{S} / \mathrm{Zn}=0.37$ compositions have much lower carrier concentration, $5 \times 10^{16} \mathrm{~cm}^{-3}$, than the $\mathrm{S} / \mathrm{Zn}=0.14$ and 0.26 compositions, $10^{19} \mathrm{~cm}^{-3}$. Although the carrier concentration for $\mathrm{S} / \mathrm{Zn}$ $=0.5$ composition has not been measured in the work, it is expected to be comparable to or lower than that for the $\mathrm{S} / \mathrm{Zn}=0.37$ composition. As one transitions from $\mathrm{ZnO}$ to $\mathrm{ZnS}$, the difficulty of $n$-type doping through intrinsic or extrinsic defects increases [33].

Using X-ray photoelectron spectroscopy (XPS) and UV-vis spectrophotometry, we measure a conduction band offset of $-0.38 \pm 0.2 \mathrm{eV}$, at the $\mathrm{SnS} / \mathrm{Zn}(\mathrm{O}, \mathrm{S})$ interface for $\mathrm{S} / \mathrm{Zn}=0.14$. The conduction band offset for $\mathrm{Zn}(\mathrm{O}, \mathrm{S})$ with $\mathrm{S} / \mathrm{Zn}=0.23$ and $\mathrm{SnS}$ was determined to be $0 \pm 0.2 \mathrm{eV}$. We anticipate the band offset of the $\mathrm{S} / \mathrm{Zn}=0.23$ composition to be very close to that of the $\mathrm{S} / \mathrm{Zn}=0.26$ composition used in devices. The conduction band offset for $\mathrm{Zn}(\mathrm{O}, \mathrm{S})$ with $\mathrm{S} / \mathrm{Zn}=0.36$ and $\mathrm{SnS}$ was determined to be $+0.24 \pm 0.2 \mathrm{eV}$. See Methods section for details of XPS interpretation. The conduction band offset for $\mathrm{S} / \mathrm{Zn}=0.5$ was taken from Ref. [34. The increasing conduction band offset with $\mathrm{S} / \mathrm{Zn}$ composition is consistent with previous reports [1], 12].

In Figure 4b we compare experimental (solid) and simulated (dashed) current-voltage curves for the characterized buffer layer composition indicated in Figure 4 a. The simulated curves for each buffer layer composition can vary significantly within the measured errobars in Figure 4 a , but only those best matching the data are shown. The S/Zn $=0.14$ with nitrogen doping has the highest experimental performance (shown in maroon). As indicated in Figure [4a, this buffer layer has low carrier concentration (between $10^{15}$ and $\left.10^{16} \mathrm{~cm}^{-3}\right)$ and a cliff $(-0.38 \mathrm{eV})$. The interface recombination velocity assumed in Figure $4 \mathrm{a}\left(2 \times 10^{3} \mathrm{~cm} / \mathrm{s}\right)$ is found by fitting the $V_{\mathrm{OC}}$ point to this device (See Methods for details). Buffer layers with $\mathrm{S} / \mathrm{Zn}=0.37$ and 0.5 show lower $J_{\mathrm{SC}}$, consistent with current blocking seen 
a

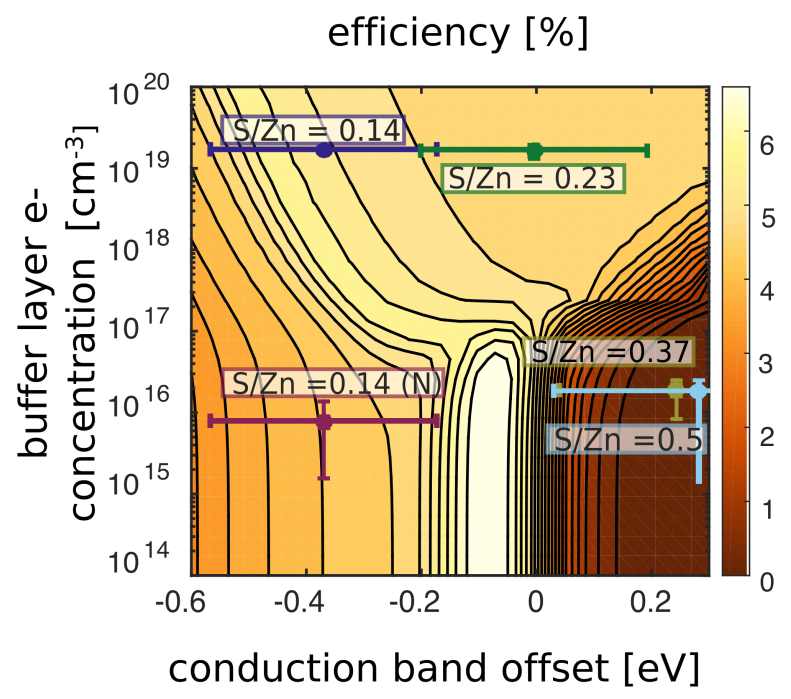

b

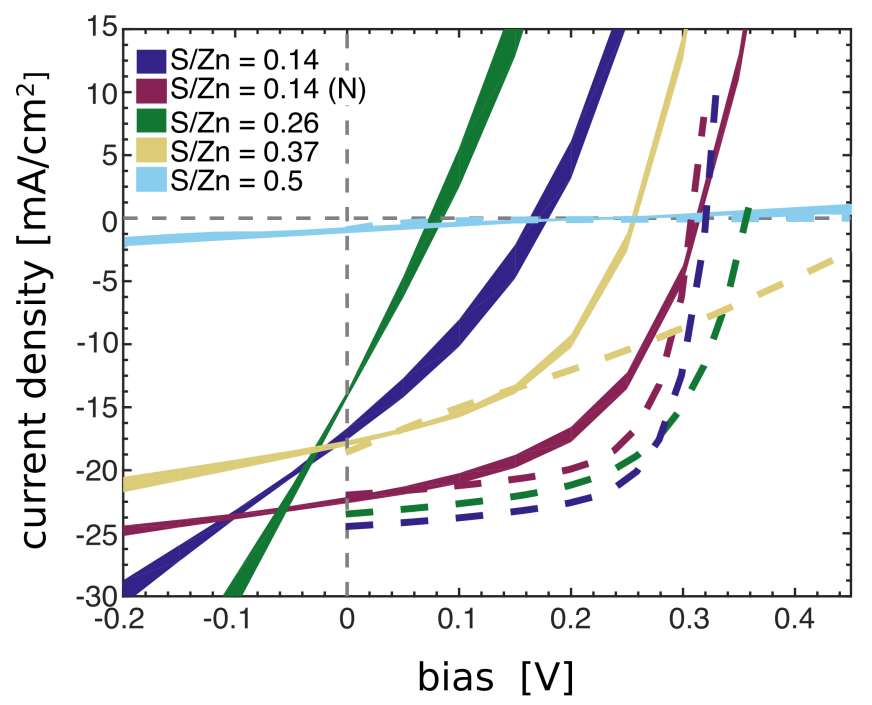

FIG. 4. Contact-layer performance space for $\mathrm{Zn}(O, S)$ buffer layers in present-day scenario: Subplot (A) shows the efficiency for varying $\mathrm{Zn}(\mathrm{O}, \mathrm{S})$ buffer layers in $\mathrm{SnS}$ devices with $100 \mathrm{ps}$ lifetime, optical losses and other loss mechanisms characteristic of present-day devices. Color indicates high (lighter) and low (darker) efficiency from 0 to $7 \%$. The dots and error bars indicate conduction band offset, as measured by XPS, and buffer layer carrier concentrations, as measured by resistivity, for Zn(O,S) buffer layers for $\mathrm{S} / \mathrm{Zn}=0.14,0.26,0.37$, and $0.5 . \mathrm{S} / \mathrm{Zn}=0.14$ is shown with and without nitrogen doping. Subplot (B) show the experimental (solid) and simulated (dashed) current-voltage results of SnS devices with the same set of Zn(O,S) buffer layers as indicated in subplot (A). The area of each experimental (solid) current-voltage curve indicates the standard error across non-shunted devices grown on the same substrate, except for $\mathrm{S} / \mathrm{Zn}=0.5$ which shows the total spread across devices on single substrate.

in the simulated curves. The discrepancy in $V_{\mathrm{OC}}$ for $\mathrm{S} / \mathrm{Zn}=0.37$ (gold) can be attributed to shunt resistance (50-200 $\Omega \mathrm{cm}^{2}$ ) in experimental devices, which is not included in this simulation. Despite the prediction of $4-5 \%$ for $\mathrm{S} / \mathrm{Zn}$ $=0.14$ and 0.26 , the experimental current-voltage curves are completely shunted.

\section{DISCUSSION}

Using a computationally fast 1-D model, we evaluate a vast performance space for the contact-layer (or buffer layer) $\mathrm{Zn}(\mathrm{O}, \mathrm{S})$ on SnS. With these performance spaces, we can easily identify the contact-layer carrier concentration and conduction band-offset resulting in the highest efficiency device. For the high-lifetime simulations in Figure 3 , the results are consistent with simulation results for other material systems [1. However, the 2-D contour map provides a more complete roadmap for experimental exploration. The simulations for the present day scenario are largely consistent with experiment for low-carrier-concentration buffer layers, while devices with high-carrier-concentration buffer layers show shunting not captured in simulation.

\section{A. Simulations can provide a comprehensive mapping of contact-layer performance to guide experiment}

These performance space plots help guide experiments through two challenges in optimizing contact-layers: (1) navigating the non-monotonic complex behavior in contact-layer performance space for a specific abosorber and (2) re-optimizing the contact-layer as the absorber lifetime improves. The performance space for a given absorber can be very complex; Figures 3 and 4 both show oddly shaped maxima and minima. Completely resolving the behavior in such a complex space through experiment alone would require the fabrication of a large number of devices. Indeed, the data points for experimental optimization in Figure $4 \mathrm{p}$ do not cover the maxima of this performance space. Additionally, relying on local behavior could be deceiving. For example, if a series of experiments found the local 
maxima in Figure 3 at the conduction band offset of $-0.1 \mathrm{eV}$ and carrier concentration of $<5 \times 10^{16} \mathrm{~cm}^{-3}$ small changes in either band-alignment or carrier concentration would result in a decrease in efficiency, discouraging further exploration in those directions and missing valuable efficiency gains. However, simulation allows a more comprehensive view of the performance space, potentially streamlineing experimental efforts and exposing local and global maxima.

The second way that performance space plots can help guide device optimization is by identifying changes in location of the global maxima as other material layers in the device are improved. As we move toward high-efficiency SnS devices, Figure 4 and Figure 3 can be used to re-optimize the contact-layer as the position of the global maxima within the performance space changes.

\section{B. Relative efficiency of high and low carrier concentration maxima depend on magnitude of bulk and} interface recombination

In our comparison between present-day and high-efficiency SnS devices, the absolute maximam of the performance space moves from the low carrier concentration maximam to the high carrier concentration maximam. In the presentday scenario there is not a clear high carrier concentration maxima, but we will refer to the maxima locations predicted by the high-efficiency scenario for both scenarios. The change in location of the global maxima is directly due to changes in bulk lifetime. A comparison of the interface and bulk recombination at the location of the two maxima in the present-day scenario are shown in Table II The higher buffer layer carrier concentration suppresses interface recombination by an order of magnitude $\left(0.55\right.$ to $\left.0.025 \mathrm{~mA} / \mathrm{cm}^{2}\right)$. As the buffer layer carrier concentration increases, the location of the $p$ - $n$ junction (or equal electron and hole concentrations) moves from the interface into the bulk, increasing bulk recombination by $21 \mathrm{~mA} / \mathrm{cm}^{2}$. For devices with relatively low interface recombination velocities of $2 \times 10^{3} \mathrm{~cm} / \mathrm{s}$ and relatively high bulk recombination, the increase in bulk recombination outweighs the suppression of interface recombination velocity. At higher interface recombination velocities, the low buffer-layer carrier concentration maximum would be less advantageous. As we move to higher-lifetime SnS materials, a few $\mathrm{mA} / \mathrm{cm}^{2}$ interface recombination current will become substantial compared to the bulk recombination and the high carrier concentration maximum will be more advantageous.

\begin{tabular}{|c|c|c|}
\hline \multicolumn{3}{|c|}{ Bulk and interface recombination at each maximum in the present-day scenario } \\
\hline & Low carrier concentration maximum & high carrier concentration maximum \\
\hline conduction band-offset & $-0.1 \mathrm{eV}(\mathrm{cliff})$ & $0.1 \mathrm{eV}$ (spike) \\
\hline buffer carrier concentration & $10^{15} \mathrm{~cm}^{-3}$ & $10^{19} \mathrm{~cm}^{-3}$ \\
\hline \hline bulk recombination & $11 \mathrm{~mA} / \mathrm{cm}^{2}$ & $32 \mathrm{~mA} / \mathrm{cm}^{2}$ \\
\hline interface recombination & $0.55 \mathrm{~mA} / \mathrm{cm}^{2}$ & $0.025 \mathrm{~mA} / \mathrm{cm}^{2}$ \\
\hline
\end{tabular}

TABLE II. Bulk $\left(J_{\text {bulk }}(V)\right)$ and interface recombination $\left(J_{\text {interface }}(V)\right)$ currents at the the two maximum from Figure 3 for the present-day scenario shown in Figure 4 . The total current out of the device can be written as a linear combination of the illumination current and all the recombination currents: $J_{\text {total }}(V)=J_{\text {illumination }}-J_{\text {bulk }}(V)-J_{\text {interface }}(V)-J_{\text {other }}(V)$. Each recombination term in this equation is the integration of the numerically calculated recombination in the device simulation over the relevant region at a particular voltage. The bias is $0.36 \mathrm{~V}$.

\section{Robust modeling: sensitivity to interface recombination velocity and other variables}

As described in the Methods section, the interface recombination velocity is the only fitting parameter in our model. As of yet, no independent experimental evaluation of our prediction of an interface recombination velocity of $2 \times 10^{3}$ $\mathrm{cm} / \mathrm{s}$ exists. To explore the impact of interface recombination velocity we simulated the buffer layer design space plots for our high-efficiency (Figure S4a-c) and present-day (Figure S5a-c) devices with interface recombination 500, 2000, and $10000 \mathrm{~cm} / \mathrm{s}$. 500 and $10000 \mathrm{~cm} / \mathrm{s}$ represent the limits of the interface recombination velocities which fit the experimental data, as described in the methods section. The qualitative features of the design space plots do not change with varying absorber-buffer interface recombination. In the high-efficiency case the two local maxima still exist, and their positions are minimally altered by increasing interface recombination. In both scenarios the region with absorber-buffer conduction-band-offset between -0.2 and $0.2 \mathrm{eV}$ and buffer-layer carrier concentration greater than $10^{17} \mathrm{~cm}^{-3}$ are unaffected. For further discussion of these simulations see the supplemental material.

The relative efficiency and location of the two local maxima also depend on all the parameters that set the builtin voltage in the device, including density of states, relative band alignment, carrier concentrations, and dielectric 
constants. For example, a sensitivity analysis at the low carrier concentration maximum in Figure 4 a, finds the efficiency at this point changes by $7 \%$ relative to the baseline simulation if the SnS static permittivity varies within literature values (13.7 to 50) [3, 19]. Varying the $\operatorname{SnS~} h^{+}$carrier density from $5 \times 10^{15} \mathrm{~cm}^{-3}$ to $5 \times 10^{16} \mathrm{~cm}^{-3} \mathrm{changes}$ the efficiency by $22 \%$ relative to the baseline simulation at the same point. Despite this sensitivity, the qualitative behaviors, such as the current blocking region and cliff type offset/low carrier concentration, are robust to variations.

\section{Practical implications and strategies for optimizing device design}

Our simulations for the present-day scenario in Figure 4 indicate that even with increased bulk recombination, high buffer-layer carrier concentrations should yield high-performance devices compared to the experimentally best performing device with $\mathrm{S} / \mathrm{Zn}=0.14$ and nitrogen doping. However, for both high buffer-layer carrier concentration devices $\left(\mathrm{S} / \mathrm{Zn}=0.14\right.$ and 0.26 without nitrogen doping), we experimentally observe shunted devices with low $V_{\mathrm{OC}}$ 's. This behavior is consistent with presence of pinholes, cracks or voids in our absorber layers. The 1-D nature of the simulation inherently assumes that films are uniform and cannot capture such heterogeneities. Pinholes can be coated with the buffer-layer material during highly conformal atomic layer deposition. If the buffer layer is highly doped, it will create a highly conductive pathway between the emitter and back contact metal, resulting in low shunt resistances. Low-carrier concentration buffer layers with slight cliff $(-0.1 \mathrm{eV})$ offsets may be necessary until these cracks can be eliminated during processing.

Taken together, this work suggests two approaches to contact- or buffer layer optimization as defined by the two maxima in Figures 3 and 4. Approach (1) is to target the high-carrier concentration maximum (location A in Figure 3) by achieving buffer layer doping levels at or greater than $10^{19} \mathrm{~cm}^{-3}$. Below carrier concentrations of $10^{18} \mathrm{~cm}^{-3}$ current blocking causes efficiencies to drop precipitously. This region represents the highest achievable efficiencies. As bulk lifetime of $\mathrm{SnS}$ (or any absorber material) increases, efficiency benefits will be most noticeable and measurable in this region. Additionally, performance in this region is robust to a wide range of conduction band offset between -0.15 and $0.3 \mathrm{eV}$. The conduction band of $\mathrm{SnS}$ varies with grain orientation by more than $100 \mathrm{mV}$ [35], so insensitivity to this variation is advantageous. Encouragingly, our current experimental buffer layer band offset and carrier concentrations suggest that we can fabricate $\mathrm{Zn}(\mathrm{O}, \mathrm{S})$ buffer layers in this region using the $\mathrm{S} / \mathrm{Zn}=0.26$ composition. However, success requires that we overcome the shunting problems in our present-day SnS absorber layer.

As many early-stage thin films may suffer from non-uniform growth, cracks, or pinholes, targeting the low-carrier concentration maximum (point B in Figure 3) may be a useful approach during absorber growth optimization. In this case, approach (2), the buffer layer carrier concentration may vary between $10^{14}$ and $5 \times 10^{16} \mathrm{~cm}^{-3}$ without affecting device performance. However, this approach is very sensitive to the conduction band offset, and only a narrow range $0.1 \mathrm{eV}$ wide around a $-0.1 \mathrm{eV}$ cliff have high efficiencies. While this approach should support efficiencies greater than $10 \%$, it will eventually limit efficiency improvement and a transition to approach (1) is desirable. To realize approach (2) nitrogen doping could be used on a $\mathrm{Zn}(\mathrm{O}, \mathrm{S})$ layer with composition between $\mathrm{S} / \mathrm{Zn}=0.14$ and 0.26 to achieve -0.1 $\mathrm{eV}$ conduction band alignment. Regardless of the path chosen, we demonstrate that current buffer layer deposition technology can deposit $\mathrm{Zn}(\mathrm{O}, \mathrm{S})$ layers with conductivity and conduction band offsets suitable for either approach.

\section{CONCLUSION}

Here we present a framework to identify a suitable charge-carrier contact layer for photovoltaic absorber materials. Our analysis of device performance with a 2-D contour plot provides clear bounds for the conduction band offset and buffer-layer carrier concentration that will provide the highest efficiency device for a given absorber. We demonstrate the application of this performance space for devices with an $\mathrm{SnS}$ absorber layer and $\mathrm{Zn}(\mathrm{O}, \mathrm{S})$ buffer layer as the contacting layer. Analysis of a high-efficiency scenario reveal two local maxima in the performance space. The first local maximum, which supports efficiencies above $16 \%$, occurs for buffer carrier concentrations less than $10^{16} \mathrm{~cm}^{-3}$ and a narrow range of conduction band offsets ( 0 to $-0.1 \mathrm{eV}$ cliff). The second maximum, which supports efficiencies above $20 \%$, occurs at buffer-layer carrier concentrations of greater than $10^{18} \mathrm{~cm}^{-3}$ and a broad range of conduction band offsets (-0.2 eV cliff to $0.2 \mathrm{eV}$ spike). These two maxima represent different approaches for buffer-layer optimization.

The performance space for a present-day scenario, representative of current bulk minority carrier lifetimes and other losses, shows that the maximum at low buffer-layer carrier concentrations has the highest efficiency. For devices with $100 \mathrm{ps}$ lifetime and $10^{2} \mathrm{~cm} / \mathrm{s}$ interface recombination velocity, a high-carrier-concentration buffer layer actually increases defect-assisted recombination in the absorber and the effect of decreasing interface recombination is negligible. Experimental characterization of conduction-band offsets and buffer-layer carrier concentrations for $\mathrm{Zn}(\mathrm{O}, \mathrm{S})$ shows that all regimes, including both maxima, of the buffer-layer performance space are accessible using the $\mathrm{Zn}(\mathrm{O}, \mathrm{S})$ buffer-layer system. Experimental device performance for buffer layers with low buffer-layer carrier 
concentrations are consistent with those predicted by theory, while devices with high carrier-concentration bufferlayers show shunting.

Performance plots such as these could be used to rapidly optimize buffer layer pairings for any material assuming the rest of the device is reasonably well characterized. Additionally, this work suggests a generalizable approach to buffer-layer pairings throughout material development and may help explain the range of variation in empiricallyoptimized device architectures in literature. During early stage material development, the lower carrier-concentration maximum is ideal, as it is robust to shunts and low minority-carrier lifetimes often present in these materials. Once uniform, shunt-free films with higher minority carrier lifetimes are achieved, the higher carrier-concentration can be targeted to support efficiencies approaching the Shockley-Queisser limit.

\section{ACKNOWLEDGEMENTS}

N.M.M., R.E.B., R.J., and T.B. designed the study. N.M.M. and R.E.B. developed the model with parameter estimation from R.J. and R.C.. N.M.M. carried out and analyzed the simulations. C.Y. deposited and characterized the buffer layers with XPS, Hall effect, resistivity, and optical measurements. H.H.P., C.Y. and X.Z. deposited buffer layers for devices. J.R.P., R.E.B, and R.J. designed and performed photoconductivity experiments. R.E.B. analyzed XPS results. V.S., R.J., and R.C. fabricated devices. V.S. and J.R.P. performed cell electrical measurements. N.M.M., R.E.B., V.S., R.J. and T.B. wrote the manuscript. T.B. and R.G. supervised this research.

The authors are thankful to Mariela Lizet Castillo for substrate preparation, Dr. Katy Hartman and Alexander Polizzotti for annealing SnS films and for scientific discussion, and Oki Gunawan at IBM for assistance with lock-in Hall effect measurements. This work is supported by the U.S. Department of Energy through the SunShot Initiative under contract DE-EE0005329, and by the National Science Foundation Division of Electrical, Communications and Cyber Systems under grant number 1102050. V. Steinmann, R. Jaramillo, and R. E. Brandt acknowledge the support of, the Alexander von Humboldt foundation, a DOE EERE Postdoctoral Research Award, and an NSF GRFP Fellowship respectively. This work made use of the Center for Materials Science and Engineering at MIT which is supported by the National Science Foundation (NSF) under award DMR-08-19762, and the Center for Nanoscale Systems at Harvard University which is supported by NSF under award ECS-0335765.

[1] Alex Niemegeers, Marc Burgelman, and Alexis De Vos. On the CdS/CuInSe2 conduction band discontinuity. Applied Physics Letters, 67(6):843, 1995.

[2] T. Unold and H.W. Schock. Nonconventional (Non-Silicon-Based) Photovoltaic Materials. Annual Review of Materials Research, 41:297-321, 2011.

[3] Julien Vidal, Stephan Lany, Mayeul D'Avezac, Alex Zunger, Andriy Zakutayev, Jason Francis, and Janet Tate. Bandstructure, optical properties, and defect physics of the photovoltaic semiconductor SnS. Applied Physics Letters, 100(3):032104, 2012.

[4] K. T. Ramakrishna Reddy, N. Koteswara Reddy, and R. W. Miles. Photovoltaic properties of SnS based solar cells. Solar Energy Materials and Solar Cells, 90:3041-3046, 2006.

[5] Hidenori Noguchi, Agus Setiyadi, Hiromasa Tanamura, Takao Nagatomo, and Osamu Omoto. Characterization of vacuumevaporated tin sulfide film for solar cell materials. Solar Energy Materials and Solar Cells, 35:325-331, 1994.

[6] Vera Steinmann, R. Jaramillo, Katy Hartman, Rupak Chakraborty, Riley E. Brandt, Jeremy R. Poindexter, Yun Seog Lee, Leizhi Sun, Alexander Polizzotti, Helen Hejin Park, Roy G. Gordon, and Tonio Buonassisi. 3.88\% Efficient tin sulfide solar cells using congruent thermal evaporation. Advanced Materials, pages 7488-7492, 2014.

[7] M. Vasudeva Reddy, G. Sreedevi, Chinho Park, R.W. Miles, and Ramakrishna Reddy. Development of Sulphurized SnS Thin Film Solar Cells. Current Applied Physics, 2015.

[8] Prasert Sinsermsuksakul, Leizhi Sun, Sang Woon Lee, Helen Hejin Park, Sang Bok Kim, Chuanxi Yang, and Roy G. Gordon. Overcoming Efficiency Limitations of SnS-Based Solar Cells. Advanced Energy Materials, 4(15):1400496, June 2014.

[9] Susanne Siebentritt. What limits the efficiency of chalcopyrite solar cells? Solar Energy Materials and Solar Cells, 95(6):1471-1476, June 2011.

[10] Prasert Sinsermsuksakul, Katy Hartman, Sang Bok Kim, Jaeyeong Heo, Leizhi Sun, Helen Hejin Park, Rupak Chakraborty, Tonio Buonassisi, and Roy G. Gordon. Enhancing the efficiency of SnS solar cells via band-offset engineering with a zinc oxysulfide buffer layer. Applied Physics Letters, 102(5):053901, 2013.

[11] Clas Persson, Charlotte Platzer-Björkman, Jonas Malmström, Tobias Törndahl, and Marika Edoff. Strong valence-band offset bowing of ZnO1-xSx enhances p-type nitrogen doping of ZnO-like alloys. Physical Review Letters, 97(October):1-4, 2006.

[12] Ryosuke Hamazaki, Takashi Minemoto, Yusuke Oda, Shohei Fukamizu, Akito Yamamoto, and Hideyuki Takakura. Op- 
timization of compositional ratio of $\mathrm{Zn}(\mathrm{O}, \mathrm{S})$ window layer in CuInS 2 solar cells. Japanese Journal of Applied Physics, $51: 2-5,2012$.

[13] Helen Hejin Park, Rachel Heasley, Leizhi Sun, Vera Steinmann, Rafael Jaramillo, Katy Hartman, Rupak Chakraborty, Prasert Sinsermsuksakul, Danny Chua, Tonio Buonassisi, and Roy G Gordon. Co-optimization of SnS absorber and $\mathrm{Zn}(\mathrm{O}, \mathrm{S})$ buffer materials for improved solar cells. Progress in Photovoltaics: Research and Applications, 23(7):901-908, 2014.

14] Marc Burgelman, Johan Verschraegen, Stefaan Degrave, and Peter Nollet. Modeling thin-film PV devices. Progress in Photovoltaics Research and Applications, 12(23):143-153, March 2004.

[15] Jiaxiong Xu and Yuanzheng Yang. Study on the performances of SnS heterojunctions by numerical analysis. Energy Conversion and Management, 78:260-265, 2014.

[16] Niall M. Mangan, Riley E. Brandt, Vera Steinmann, R. Jaramillo, Jian V. Li, Jeremy R. Poindexter, Katy Hartman, Leizhi Sun, Roy G. Gordon, and Tonio Buonassisi. A path to $10 \%$ efficiency for tin sulfide devices. In Proceedings of the 40th IEEE Photovoltaic Specialist Conference, pages 2373-2378. IEEE, June 2014.

[17] R. Jaramillo and Et al. THz-based minority carrier lifetime measurements on SnS thin films: Advanced metrology for an early-stage photovoltaic material. In Preperation, 2015.

[18] Rafael Jaramillo, Meng-Ju Sher, Ben Ofori-Okai, Vera Steinmann, Katy Hartman, Keith A Nelson, Aaron Lindenberg, and Tonio Buonassisi. Minority-Carrier Lifetime Measurements on Tin Sulfide Thin Films Using THz Free-Carrier Absorption. In Materials Research Society Meeting, 2015.

[19] R. E. Banai, L. A. Burton, S. G. Choi, F. Hofherr, T. Sorgenfrei, A. Walsh, B. To, A. Cröll, and J. R S Brownson. Ellipsometric characterization and density-functional theory analysis of anisotropic optical properties of single-crystal $\alpha$-SnS. Journal of Applied Physics, 116:013511, 2014.

[20] Rafael Jaramillo, Vera Steinmann, Chuanxi Yang, Katy Hartman, Rupak Chakraborty, Jeremy R. Poindexter, Mariela Lizet Castillo, Roy Gordon, Tonio Buonassisi, and M. L. Castilo. Making record-efficiency SnS solar cells by thermal evaporation and atomic layer deposition. J. Vis. Exp., 99(99):e52705, May 2015.

[21] N. Ashkenov, B. N. Mbenkum, C. Bundesmann, V. Riede, M. Lorenz, D. Spemann, E. M. Kaidashev, A. Kasic, M. Schubert, M. Grundmann, G. Wagner, H. Neumann, V. Darakchieva, H. Arwin, and B. Monemar. Infrared dielectric functions and phonon modes of high-quality ZnO films. Journal of Applied Physics, 93(1):126-133, 2003.

[22] S. Zh Karazhanov, P. Ravindran, a. Kjekshus, H. Fjellvå g, U. Grossner, and B. G. Svensson. Coulomb correlation effects in zinc monochalcogenides. Journal of Applied Physics, 100(2006), 2006.

[23] V Quemener, M Alnes, L Vines, P Rauwel, O Nilsen, H Fjellvå g, E V Monakhov, and B G Svensson. The work function of n-ZnO deduced from heterojunctions with Si prepared by ALD. Journal of Physics D: Applied Physics, 45:315101, 2012.

[24] Chuanxi Yang, Leizhi Sun, Riley E. Brandt, Xizhu Zhao, Jun Feng, Tonio Buonassisi, and Roy G. Gordon. nvestigating Contact Resistivity at Metal-Tin Sulfide (SnS) Interfaces. In Preperation, 2015.

[25] Rupak Chakraborty, Vera Steinmann, Niall M. Mangan, Riley E. Brandt, Jeremy R. Poindexter, Rafael Jaramillo, Jonathan P. Mailoa, Kathrine Hartman, Alexander Polizzotti, Chuanxi Yang, Roy G. Gordon, and Tonio Buonassisi. Nonmonotonic effect of growth temperature on carrier collection in SnS solar cells. Applied Physics Letters, 106(20):203901, 2015.

[26] B. Hoex, J. Schmidt, P. Pohl, M. C. M. van de Sanden, and W. M. M. Kessels. Silicon surface passivation by atomic layer deposited Al2O3. Journal of Applied Physics, 104(4):044903, August 2008.

[27] Baochen Liao, Bram Hoex, Armin G. Aberle, Dongzhi Chi, and Charanjit S. Bhatia. Excellent c-Si surface passivation by low-temperature atomic layer deposited titanium oxide. Applied Physics Letters, 104(25):253903, 2014.

[28] O Gunawan and T Gokmen. Hall Measurement System with Rotary Magnet, 2014.

[29] Roland Scheer and HW Schock. Chalcogenide photovoltaics: Physics, Technologies, and Thin Film Devices. WILEY-VCH Verlag GmbH \& Co. KGaA, Weinheim, Germany, 1 edition, 2011.

[30] Charlotte Platzer-Björkman, Adam Hultqvist, Jonas Pettersson, and Tobias Törndahl. Band gap engineering of ZnO for high efficiency CIGS based solar cells. Proceeding of SPIE, 7603:76030F-76030F-9, 2010.

[31] J Pettersson, C Platzer-Björkman, A Hultqvist, U Zimmermann, and M Edoff. Measurement of photo-induced changes in the conduction properties of ALD-Zn(1-z) Mg(x)O thin films. Physica Scripta, T141:014010, 2010.

[32] R. Pietruszka, G. Luka, K. Kopalko, E. Zielony, P. Bieganski, E. Placzek-Popko, and M. Godlewski. Photovoltaic and photoelectrical response of n-ZnO/p-Si heterostructures with $\mathrm{ZnO}$ films grown by an Atomic Layer Deposition method. Materials Science in Semiconductor Processing, 25:190-196, 2014.

[33] S B Zhang. The microscopic origin of the doping limits in semiconductors and wide-gap materials and recent developments in overcoming these limits: a review. Journal of Physics: Condensed Matter, 14(34):R881-R903, September 2002.

[34] Leizhi Sun, Richard Haight, Prasert Sinsermsuksakul, Sang Bok Kim, Helen H. Park, and Roy G. Gordon. Band alignment of $\mathrm{SnS} / \mathrm{Zn}(\mathrm{O}, \mathrm{S})$ heterojunctions in SnS thin film solar cells. Applied Physics Letters, 103(18):181904, 2013.

[35] Vladan Stevanović, Katy Hartman, R. Jaramillo, Shriram Ramanathan, Tonio Buonassisi, and Peter Graf. Variations of ionization potential and electron affinity as a function of surface orientation: The case of orthorhombic SnS. Applied Physics Letters, 104(21):211603, May 2014. 